\title{
Los "priles" son de primera elección en la Insuficiencia Cardíaca frente a los "sartanes"
}

Effects of losartan and captopril on mortality and morbidity in high-risk patients after acute myocardial infarction: the OPTIMAAL randomised trial. Dickstein K, Kjekshus J, and the OPTIMAAL Steering Committee, for the OPTIMAAL Study Group. Lancet. 2002 Sep 7;360:752-60.

\section{Objetivo}

Comparar los efectos de losartan y captopril sobre la mortalidad en pacientes con insuficiencia cardíaca (IC) post infarto agudo de miocardio (IAM).

\section{Diseño}

Estudio multicéntrico aleatorizado, doble ciego y controlado.

\section{Lugar}

Participaron 329 centros de siete países de Europa.

\section{Pacientes}

Se incluyeron 5.477 pacientes mayores de 50 años con IAM confirmado acompañado de signos o síntomas de IC o de signos de disfunción ventricular durante la fase aguda.

\section{Intervención}

Los pacientes fueron aleatorizados dentro de los 10 días post IAM a recibir dosis progresivas hasta alcanzar $50 \mathrm{mg}$ de losartan una vez por día o $150 \mathrm{mg}$ de captopril divididas en tres tomas por día.

\section{Medición de resultados principales}

El punto final primario fue la mortalidad por tódas las causas. Los puntos finales secundarios y terciarios fueron muerte súbita o paro cardíaco resucitado y reinfarto fatal o no fatal respectivamente. El estudio fue analizado por intención de tratar.

\section{Resultados principales}

El seguimiento medio fue de 2,7 años.

Tabla 1. Resultados principales

\begin{tabular}{|c|c|c|c|c|}
\hline Puntos finales & $\begin{array}{l}\text { Losartan } \\
(n=2744)\end{array}$ & $\begin{array}{l}\text { Captopril } \\
(n=2733)\end{array}$ & $\begin{array}{c}\text { RR } \\
\text { (IC 95\%) }\end{array}$ & \\
\hline Mortalidad por todas las causas & $499(18,2 \%)$ & $447(16,4 \%)$ & $1,13(0,99-1,28)$ & 0,069 \\
\hline $\begin{array}{l}\text { Muerte súbita o paro } \\
\text { cardíacu resucitado }\end{array}$ & $239(8,7 \%)$ & 203 & $1,19(0,99-1,43)$ & 0,072 \\
\hline Reinfarto fatal o no fatal & $384(14,0 \%)$ & $379(13,9 \%)$ & $1,03(0,89-1,18)$ & 0,722 \\
\hline
\end{tabular}

No se encontraron diferencias en la tasa de hospitalización por cualquier causa entre los grupos. El losartan fue significativamente mejor tolerado que el captopril con menor número de suspensiones del tratamiento: Losartan $458(17 \%)$ y captopril $624(23 \%)$, RR 0,70 [IC $95 \%$ 0,62-0,79], $p<0,0001$

\section{Conclusiones}

El losartan no fue superior al captopril para reducir la mortalidad de los pacientes con IC post IAM.

Los pacientes tratados con losartan presentaron menos efectos adversos que los obligara a suspender el tratamiento.

Fuente de financiamiento: Merck, Sharp and Dohme Research Laboratories, West Point, EEUU

\section{Comentario}

Los pacientes con IC post IAM constituyen un grupo de altísimo riesgo de morbimortalidad. En las últimas décadas, a partir de los múltiples estudios que han demostrado los beneficios clínicos de los inhibidores de la enzima convertidora de angiotensina (IECA) incluyendo una reducción de la mortalidad, ${ }^{1}$ surgieron nuevos estudios con la hipótesis de que a través de los antagonistas de los receptores de la angiotensina II (ARA-II) podría obtenerse un bloqueo más completo y así tendrían ventajas potenciales. ${ }^{2}$

Previo al OPTIMAAL, el estudio ELITE II, diseñado para comparar la sobrevida entre losartan y captopril en pacientes con IC y fracción de eyección $<40 \%$, observó tras 1,5 años que no existe una diferencia significativa de la mortalidad entre ambos grupos: Losar$\tan (17,7 \%)$ vs Captopril $(15,9 \%)$, RR 1,13 [IC 95\% 0,95-1,35], $\mathrm{p}=0,16{ }^{3}$, Un meta-análisis de 17 ensayos clínicos aleatorizados y controlados comparó en 12.469 pacientes la mortalidad o la tasa de hospitalización entre ARA-II vs placebo o IECA en pacientes con IC en clase funcional II-IV. En este análisis no hubo diferencias significativas entre el grupo de ARA-II y el grupo control tanto para la mortalidad (OR 0,96; IC $95 \% 0,75-1,23$ ) como para la hospitalización (OR 0,86; IC 95\% 0,69-1,06).

Sin embargo, en el análisis estratificado, en ausencia de tratamiento concomitante con IECA, se observó una tendencia no significativa a favor del ARA-II sobre el placebo en la reducción de la mortalidad (OR 0,68; IC 95\% 0,38-1,22) y en la hospitalización (OR 0,67; IC 95\% 0,29-1,51). Cuando se compararon directamente con Ios IECA, Ios ARA-II no fueron superiores en la reducción de la mortalidad (OR 1,09; IC 95\% 0,92-1,29) ni en la reducción de la hospitalización (OR 0,95; IC 95\% 0,80-1,13). En cambio, la com- binación ARA-II + IECA fue superior a IECA solo reduciendo la hospitalización (OR 0,74; IC 95\% 0,64-0,86) pero no la mortalidad (OR 1,$04 ;$ IC95\% 0,91-1,20). ${ }^{4}$

Efectuamos un meta-análisis de los dos estudios mayores específicamente diseñados para evaluar comparativamente la mortalidad de Losartan vs Captopril: OPTIMAAL y ELITE II. EI RR de muerte de los pacientes tratados con Losartan en lugar de Captopril es de 1,11 (IC95\% 1,01-1.22; $p=0.02$ ) con un NND* (número necesario a dañar) por 1,5-2,7 años que sería de sólo 50 pacientes. Incluyendo otros estudios no diseñados para mortalidad la tendencia se mantiene aunque con significancia limítrofe y resultados heterogéneos. Los estudios de Losartan contra Placebo son sólo a 12 semanas de sequimiento aunque mostraron un RR de 0,35 (IC95\% 0,15-0,80; p=0.01; NNT=33). Este último resultado es consistente con el análisis de subgrupo de pacientes sin IECA del estudio Val-HeFT ${ }^{5}$ donde el Valsartán redujo $30 \%$ la mortalidad y $42 \%$ la morbimortalidad frente a los controles y al combinarlo a los estudios de Losartan contra Placebo el RR es 0,54 (IC95\% 0,35-0,83 $\mathrm{p}=0.005 ; \mathrm{NNT}=20)$. Existen actualmente varios estudios en marcha que comparan IECA con ARA-II o su combinación ${ }^{6}$.

Conclusión de los comentadores: Según la evidencia disponible, el losartan parece mostrar menor beneficio clínico que el captopril en los pacientes con IC. Los IECA son de primera elección en pacientes con IC por disfunción sistólica, y en el caso de intolerancia, Ios ARA-II podrían constituir una buena alternativa.

\section{Dra. Chu Yon, Yoo - Dr. Agustín Ciapponi [ Unidad de Medicina Familiar y Preventiva. Hospital Italiano de Buenos Aires ]}

\section{Referencias}

1. Flather MD, Yusuf S, Køber L, et al. Long-term ACE-inhibitor therapy in patients with heart failure or left-ventricular dysfuntion:a systematic overview of data from individual patients. Lancet.

2000;355:1575-81. 2.Sharma D, Buyse M, Pitt B, Rucinska EJ, and the Losaran 2000:85:187-92.

multiple-dose studies of losartan in heart failure. Am J Cardiol 2000,85:187-92. 3.Pitt B, Poole-Wilson PA, Segal R, et al. Effect o

vival Study ELITE II. Lancet 2000;355:1582-7.

4.Jong P, Demers C, McKelvie RS, et al. Angiotensin receptor blocker in heart failure : Meta-analysis of randomized controlled trials. J Am Coll Cardiol 2002;39:463-70.

5.Maggioni AP, Anand I, Gottlieb SO, Latini R, Tognoni G, Cohn JN. Effects of valsartan on morbidity and mortality in patients with heart failure not receiving angiotensin-converting enzyme inhibitors. J Am Coll Cardiol. 2002;40(8):1414.

6.Yusuf S. From the HOPE to the ONTARGET and the TRANSCEND studies: challenges in improving prognosis. Am J Cardiol 2002;89(2A):18A-25A 